\title{
The Architecture of Determiners
}

\author{
By Thomas Leu
}

New York: Oxford University Press, 216 pages. ISBN 978-0-19-994524-5

Received: 26-09-17

Accepted: 27-12-17

Reviewed by Rut Benito Galdeano

Universitat Autònoma de Barcelona

The present book allows the reader to zoom in on the internal structure and derivation of determiners both across languages and across determiners. The work shows the structural regularities of this closed class items, as well as their morphological segmentability, in order to answer what the internal structural properties that recur in different determiners are and how their structure compare to that of other kinds of constituents.

Although the discussion focuses mainly on Swiss German and German (with occasional reference to other Germanic languages and non-Germanic languages), the theoretical implications seek to reflect universal properties of human languages.

Building on generative syntax, the author proposes that determiners (except articles) are extended adjectival projections (xAP) including a root, agreement inflection and a left-periphery. This assumption reveals us that what is often thought of as non-decomposable function words is in fact syntactically (and internally) complex in a regular way.

The book is divided in two main blocks: ' $D$-terminers' (Chapters 2-4) and 'Non- $D$-Determiners' (Chapters 5-7). The first part is dedicated to determiners with a $d$ and their inflectional morphology in structural case environments, and the second part explores the indefinite determiner ein ('a'), wh-determiners, and their case and syncretism. After introducing the basis of the main proposal in Chapter 1, Thomas Leu begins his discussion with definite demonstratives. Assuming that $d$-/th- is the same morpheme in articles (der in German, the in English) and demonstratives (dieser in German, that in English), Chapter 2 'Definite Demonstratives' argues that demonstratives are not atomic elements taken from the lexicon, but are syntactically composed. According to Leu, demonstratives have the complex structure of a preadjectival article $(d-/ t h-)$ which is not always overtly realized, an agreement head $\left(\mathrm{AGR}_{\mathrm{CA}}\right)$ host of strong adjectival inflection, and an adjectival deictic/anaphoric element (here or there) which remains mostly unpronounced. This xAP stays as a left peripheral specifier of the extended noun phrase (xNP):

$$
\text { [DP [xAP the/d-AGR } \mathrm{CA} \text { HERE] [D [NP ...] ] ] }
$$


Empirical evidence for this proposal is found in other languages (e.g. Scandinavian, Afrikaans, Swiss German, Chinese), in which the deictic/demonstrative adjective is sometimes overtly realized. This analysis can be further extended to other types of adjectival components such as same or other.

Chapter 3 ' $\mathrm{xAP}$ ' investigates the morphosyntax of xAPs and generalizes the proposal for demonstratives to all definite adjectival modifiers, based on data of many different languages. In this account, The big house differs from The house in that the former has an open class adjective and the latter has the closed class adjective HERE.

In addition, the author exposes the relation of the xAP to the xNP. First, the noun originates within the XAP. When the xAP merges with $n$, the noun extracts from the xAP to [Spec, $\mathrm{nP}$ ] in a way akin to extraction of the head of a relative clause. Thus, the XAP is a (reduced) relative clause to the noun (both contain a lexical layer, an inflectional layer and a left periphery with a complementizer). The xNP is built up by merging functional heads and fronting xAPs across the noun. The result is shown in (2):

$$
\left[\mathrm{xNP} \operatorname{Spec}\left[\operatorname{noun}\left[\mathrm{n}\left[\mathrm{xAP} \ldots \operatorname{adjective} t_{\text {noun }}\right]\right]\right]\right.
$$

The XAP involves various internal movements and shows diverse surface configurations depending on various factors. The author proposes that the preadjectival definite marker is a head in the XAP, and when no definite marker is merged in the XAP, AP moves to its left periphery, crossing AGR $\mathrm{CA}_{\mathrm{A}}$. This fact explains the Germanic weak/strong adjectival declension alternation and the appearance of an additional definite marker under adjectival modification in languages as Mainland Scandinavian, Colloquial Slovenian or Greek.

The discussion follows with 'Definite Quantifiers' in Chapter 4, focusing on the distributive universal determiner jeder ('every') and distributive dual determiner beide ('both') in German. Following the assumptions of the previous chapters, Leu justifies why jeder and beide are morphosyntacticallly adjectival and project XAPs as well as definite determiners. Both quantifiers share most of their properties, inherited from the syntax of ordinary adjectival phrases, and need an extra XAP internal movement, which he dubs 'Q-movement'.

Leu decomposes the distributive determiner jeder into a quantificational morpheme je-, a definiteness marker $d$ - and an agreement marker -er. Again, morphological evidence proves that the analysis can be extended to some Greek, Romance and Slavic languages. Moreover, the distributive universal determiner jeder shows that determiners have an internal syntax akin to that of relative clauses (once again): the suffixal inflection -er is an instance of (strong) adjectival agreement; $j e$ is the adjectival stem; the element $-d$ - is an adjectival article, which is the complementizer of a reduced relative.

The second part of the book is opened by Chapter 5 'Ein-determiners'. The determiners under consideration, which have their own inflectional behaviour, usually contain the formative ein (e.g. the indefinite article, the singular numeral, some of the possessive determiners and the negative determiner). However, there are determiners that have the same inflectional properties as ein (so they belong to the same group), but do not have an overt ein (e.g. possessive $i h r$ 'her'). 
This type of determiners always involve a xNP-internal operator movement, as in (3). This operator can be overtly realized, which marks featural contrasts as negation in kein ('no') or person deixis in mein ('my'), or can be unpronounced (as in indefinite articles).

$$
\mathrm{XP} \text { ein } \ldots \mathrm{t}_{\mathrm{XP}} \ldots
$$

Similarly to definite determiners, superficial differences among these type of determiners are possibly a side effect of the derivation and the phonetic presence or absence of (parts of) the XP (it sometimes consist of multiple elements) and of ein. Interestingly, the author proves the existence of two variants of ein-determiners: the INV variant and the ADJ variant, the latter featuring both inflectional $\left(\mathrm{AGR}_{\mathrm{CA}}\right)$ and derivational adjectival morphology. Once again, the author backs up his hypothesis; this time, with possessive determiners in German and Swiss German.

Comparing different languages (and thus highlighted the importance of comparative syntax), Chapter 6 'ADJ and INV Variants Among $W h$ - and $S$ Determiners' discusses the duality of ADJ and INV variants for each non- $d$-determiner, focusing on wh- and s-determiners (e.g. was 'what' and solch 'such' in German). The study reveals that this type of non- $d$-determiners are also kinds of XAPs.

The first construction discussed is was für ('what for'). Since what for is frequently used to ask for a 'kind of N', the author introduces sort as a complement of für, which sometimes remain silent, analogous to the silent adjectival element HERE of Chapter 2. The dual configuration of INV and ADJ variants furthers extends to this construction, showing its rich underlying structure.

The second type of elements analyzed are '-lch- words' (welch 'which' and solch 'such'). Leu convincingly proves that they display the two surface variants $\mathrm{ADJ}$ and INV. These determiners can surface in one of the two variants across languages: the $\mathrm{ADJ}$ variant, where the determiner exhibits (strong) adjectival agreement; and the INV variant, the derivation of which involves an inversion movement such that the determiner ends up preceding an indefinite article.

Chapter 7 'Case and Syncretism' revisits a topic set aside before: oblique case (i.e., dative and genitive). After examining visible suffixes on the determiner (and despite analyzing German data), the syntactic properties of datives and genitives are revealed. Quite clearly, the author exposes that dative and genitive have a partly distinct positional distribution from the nominative and accusative strong agreement forms and are not strong adjectival agreement.

Given the behaviour of the nonfeminine oblique 'kase' marker $-m$, the author proposes that $-s,-m,-r$ are allomorphs of an oblique Case marker OK, since they are not instances of agreement. Leu argues that dative and genitive noun phrases are the result of an intermingling of indefinite accusative noun phrases and a $\varphi$-featurally inert possessor clitic.

If the proposals of this work are right, this study is not trivial, having consequences for morphology, semantics and the lexicon. One of the assumptions that the reader can extract is that there are no syntactically atomic determiners: the complexity of determiners is product of syntactic operations, which further reveals that syntax operates in small constituents. This means that brain operates on highly decompositional assumptions, which in fact is not exclusive of language. Moreover, it seems that all determiners behave under similar derivation 
patterns, which indicates that they are ruled by UG. If so, further research with other family languages than Germanic should be carried out to support the analyses of this book.

In conclusion, this well-grounded study paves the way for further investigations about the nature of determiners, opening encouraging paths with new theoretical analyses. At the same time, it goes beyond just determiners, since it makes linguistics be a bit closer to the architecture of UG and brain operations. 